\title{
Lack of association between IL10 and TNFa gene polymorphisms and polycystic ovary syndrome in Saudi women
}

\author{
A.F. Alkhuriji ${ }^{1}$, S.Y. Al Omar ${ }^{1}$, Z.A. Babay ${ }^{2}$, M.F. El-khadragy ${ }^{1,3}$, \\ W.G. Allharbi ${ }^{1}$ and A.A. Alnafjan ${ }^{1}$ \\ ${ }^{1}$ Department of Zoology, College of Science, King Saud University, Riyadh, \\ Saudi Arabia \\ ${ }^{2}$ Department of Obstetrics and Gynaecology, King Saud University, King \\ Khaled University Hospital, Riyadh, Saudi Arabia \\ ${ }^{3}$ Department of Zoology and Entomology, College of Science, Helwan \\ University, Cairo \\ Corresponding author: A.F. Alkhuriji \\ E-mail: aalkhuriji@ksu.edu.sa \\ Genet. Mol. Res. 18 (3): gmr18270 \\ Received February 25, 2018 \\ Accepted July 01, 2019 \\ Published July 04, 2019 \\ DOI http://dx.doi.org/10.4238/gmr18270
}

\begin{abstract}
Polycystic ovarian syndrome (PCOS) is the most prevalent endocrine disorder affecting females. It is a common cause of menstrual irregularities and infertility during a woman's reproductive years. Many factors may play a crucial role in the pathogenesis of POCS. One of these factors is related to immunogenetics. Cytokines are significant immunomodulatory proteins for regulating and controlling cell functions involved in the immune system. The imbalance between pro- and anti-inflammatory pathways plays a role in PCOS etiology. We investigated the relationship between gene polymorphisms interleukin (IL-10) (rs1800871, rs1800872, rs1800896) and tumor necrosis (TNF- $\alpha$ ) (rs1799724) in Saudi women with PCOS .The study group consisted of 93 Saudi females (mean age $31.05 \pm 0.590$, range 18 - 40 years) with PCOS. The control group consisted of 98 Saudi women without PCOS. Blood was obtained, and DNA was extracted for each patient and control. Single nucleotide polymorphisms (SNPs) of promoter regions were determined using Taq Man genotyping assays. The
\end{abstract}


polymorphism frequencies in IL-10 (rs1800871) A/A, A/G, and G/G genotypes were $7.5 \%, 38.7 \%$, and $53.76 \%$, respectively in PCOS patients and $5.1 \%, 45.9 \%$, and $49.0 \%$, respectively in controls with no significant differences between the groups. No significant differences in IL-10 polymorphism frequencies in $\mathrm{C} / \mathrm{C}, \mathrm{C} / \mathrm{T}$, and $\mathrm{T} / \mathrm{T}$ between groups were noted. No significant differences were seen ple parameters for TNFbetween groups with respect to the sam TNF$\alpha$. The differences in frequencies of alleles and genotypes were not significant between Saudi women with PCOS and controls. We conclude that though in some populations, meta-analyses showed an association between IL-10 and TNF- $\alpha$ gene polymorphisms and PCOS, this association is not apparent in Saudi females.

Key words: IL-10; TNF- $\alpha$ gene polymorphism; Saudi Arabia; Polycystic ovary syndrome (PCOS)

\section{INTRODUCTION}

The most unique and habitually encountered endocrine malfunction in women is polycystic ovary syndrome (PCOS) (Qin et al., 2015; Sóter et al., 2015; Xing et al., 2017). Overproduction of androgens, ovulatory malfunctions, and other anomalies in ovarian morphology (Zhang et al., 2015) are some of the characteristics of PCOS, which is found in approximately 9-21\% women of reproductive age (Yamada et al., 2017; Zeng et al., 2018). PCOS is thought of as a biological issue that impacts including other body systems, for example, those associated with psychological structure and metabolic, and procreative systems (Talaat et al., 2016). Androgen instability as the cause of syndromes involving the hypothalamus, pituitary glands, and ovaries is one of the causes of PCOS. Elevated gonadotropin activity, leading to increased levels of luteinizing hormone (LH), which stimulates ovaries to release a greater amount of androgens, mostly testosterone, is the main cause of PCOS (Szczuko et al., 2016).

PCOS is a common disease that is ascribed to a handful of hereditary and environmental risk factors. Different PCOS phenotypes may result from the interaction between a number of predisposing genomic mutations, each exerting only minor functions, and strong environmental factors (Zhang et al., 2015). PCOS is indicate by important metabolic abnormalities that include deformities of energy use, which causes obesity $(\mathrm{Ob})$, glucose-induced hyperinsulinemia, and fasting, peripheral insulin resistance that disturbs the muscle and adipose tissue metabolism and also causes dyslipidemia (Wang et al., 2017; Zhang et al., 2018). It is frequently represented by hyperandrogenism with great risks of insulin resistance and abdominal obesity. It is also related to infertility, acne, hirsutism, infertility, increased risk of cardiovascular diseases, hypertension, high blood pressure, dyslipidemia, inflammation, and type 2 diabetes (Fulghesu et al., 2011; Lindholm et al., 2011; Qin et al., 2015; Wu et al., 2015; Talaat et al., 2016).

Many environmental issues, such as drinking alcohol, consuming food kept plastic packages, adrenal dysfunction, and obesity are involved in PCOS development (Sóter et al., 2015; Xing et al., 2017). The etiology of PCOS includes epigenetic changes and numerous genetic changes that are regarded as unclear; nonetheless, polymorphisms found in cytokine 
genes may have a significant role, particularly the possibilities for functional SNPs affecting PCOS vulnerability. Various molecular epidemiological studies have emphasized the connection of PCOS risk and cytokine (tumor necrosis factor [TNF]- $\alpha$, interleukins [IL]-1A, $-1 \mathrm{~B},-6,-10$, and -18) gene polymorphisms (Talaat et al., 2016). This study proposes that PCO-related environmental and/or epigenetic factors interact with cytokine genes (Sóter et al., 2015). In PCOS pathogenesis, immune dysregulation could play a major role (Qin et al., 2015; Talaat et al., 2016; Xing et al., 2017). Cytokines are cell-signaling protein molecules secreted by immune cells and are engaged in many intercellular communication pathways. Discrepancies comparing pro- inflammatory and anti-bacterial cytokines and chronic infections are also believed to contribute to the history and origin of PCOS (Talaat et al., 2016).

The antenatal cytokines, which include IL-10 and -1 receptor antagonists (IL1-RA), vary with PCOS-associated inflammation (Wu et al., 2015). Regulation of inflammatory syndrome results in recruitment of regulatory B cells after excretion of IL-10 (Liu et al., 2016). IL-10 is an antenatal cytokine; in addition, it suppresses immune responses. It is also involved in the regulation of immune body processes. IL-10 was originally identified in Thelper (Th) 2 cells that repress cytokine fusion in Th1 cells. IL-10 is an anti-inflammatory cytokine, during infection, it inhibits the activity of Th1 and natural killer (NK cells and macrophages, all of which are required for optimal pathogen clearance but also contribute to tissue damage (Couper et al., 2008). PCOS patients have hyperinsulinemia and insulinresistance, which seems to indicate an infective role for the illness. IL-10 gene polymorphism may add to PCOS pathogenesis (Karadeniz et al., 2008).

Studies on reproductive biology have revealed that these pro-inflammatory cytokines influence ovarian function in addition to the processes of fertilization, implantation, and ovulation in women with PCOS (Wu et al., 2015). In contrast to the entire menstrual cycle, the corpus luteum secretes immunoreactive TNF-alpha (Guo et al., 2015). Hyperexpression of adipose tissues in addition to TNF $a$ in muscles are clearly programmed for an increase in insulin resistance (IR) through reduction of tyrosine kinase activity at the site of cellular insulin production. TNFa causes hyperandrogenism (HA), promotes IR, and is also associated with follicular formation; therefore, TNFa is implicated in PCOSassociated functional changes (Thathapudi et al., 2014).

Numerous studies have shown that polymorphisms in cytokine genes are associated with PCOS. Talaat et al. (2016) conducted a study in 61 patients with PCOS and 80 healthy controls in an Egyptian population and examined the influence of IL-10 serum levels and genetic polymorphisms with respect to the risk of PCOS. They found that -819 TT and IL10 - 1082 GG genotype could be observed as a risk factors for PCOS, and IL-10 levels were meaningfully higher in standard controls compared to PCOS patients. Xing et al. (2017) reported that they observed a significant relationship between the IL-10 rs1800871 polymorphism and development of PCOS in the Chinese population.

Thathapudi et al. (2014) indicated a role for TNF- $\alpha$ in the pathological process of $\mathrm{Ob}$ in addition to IR within PCOS, and noted C850T (rs1799724) distinct phenotypes in the promoter area of the TNF $\alpha$ gene in a group 204 age-matched healthy controls and 204 PCOS patients. This study's outcome suggests that the TNF $\alpha$ system results in initiation of IR, HA, and $\mathrm{Ob}$ in PCOS patients irrespective of the discrepancies of the TNF $\alpha$ C850T (rs1799724) in the Indian women. Sóter et al. (2015) performed a study in 196 age-matched women with 97 healthy women as controls and 99 with PCOS. Using polymerase chain 
reaction (PCR), the authors were able to show a correlation between IL-10 and -6, interferon (IFN)y, and transforming growth factor (TGF)b1 distinct phenotypes associated with genes encoding inflammation-associated factors from peripheral blood-derived DNA and cytokines. They noticed that distinct gene cytokine-associated phenotypes were not found in Brazilian women with PCOS development; nevertheless, these phenotypes may result in common metabolic complaints associated with PCOS.

The association of the polymorphisms of the TNFa and IL-10 and -6 genes with PCOS occurrence and the clinical/laboratory characteristics in Swedish residents was described in detail. Vural et al. (2010) designed a study with SNPs of the TNF- $a$ (-308 G/A), IL-6 (-174 G/C), IL-10(-1082 G/A) genes in DNA 95 healthy controls and in peripheral white blood cells of 97 patients with PCOS. They showed that there was a trend toward a higher rate of normal genotype in the controls compared to the $\mathrm{C}$ allele and IL-6 $\mathrm{CC}$ genotype among PCOS women although this difference was not expected. There were no or very small difference observed between groups in allele or genotype frequencies for $\mathrm{TNF} a$ and IL-10 genes. Yun et al. (2011) performed a study with 144 healthy women as controls out of a total of 217 study patients. They did a comparative study of the -1031(T/C) genetic variation in the TNFa gene with PCOS in Korean women; they found a relationship in PCOS with the $-1031(\mathrm{~T} / \mathrm{C})$ polymorphism in the promoter region of the TNF $a$ gene $(\mathrm{P}-$ value $=0.0003$; odds ratio $[\mathrm{OR}]=2.53$ ). However, controls were smaller in number than the $\mathrm{C}$ allele $\mathrm{PCOS}$ patients.

Karadeniz et al. (2008) studied the IL-10 polymorphism in a study of Turkish women, consisting 74 healthy controls and 91 young women with PCOS. They found that the IL-10 gene polymorphism of PCOS patients was not involved with changes in inflammatory markers.

In our study, we examined the relationship between the gene polymorphisms IL-10 (rs1800871, rs1800872, rs1800896) and TNFa (rs1799724) in Saudi women with PCOS.

\section{MATERIAL AND METHODS}

\section{Subjects}

This study included 93 Saudi women (mean age $31.05 \pm 0.59$ years) who had PCOS and had presented to the King Khaled University Hospital, Riyadh, Saudi Arabia. The control group included 98 (mean age $31.48 \pm 0.49$ years) subjects. Ethical approval for the study was obtained from the Medical Ethics Committee of King Khalid University Hospital and the Ethical Committee of King Saud University. All patients and controls provided informed consent and agreed to give blood samples for this case-control study.

\section{DNA preparation}

The study group consisted of 93 Saudi females (mean age $31.05 \pm 0.590$, range 18 40 years) with PCOS, and the controls consisted of 98 non-PCOS Saudi women. Blood was obtained and genomic DNA was extracted from peripheral blood using the Puregene purification kit (Qiagen; Hilden, Germany) according to the manufacturer's protocol. Quantification of extracted DNA was performed using a NanoDrop ND-2000c spectrophotometer (Thermo Scientific, Wilmington, DE, USA). 


\section{Genotyping of IL-10 rs1800871, rs1800872, rs1800896, and TNF-a rs1799724}

Subjects were genotyped for polymorphism IL-10 rs1800871, rs1800872, rs1800896, and TNF-a rs1799724 using the TaqMan ${ }^{\circledR}$ SNP Genotyping Assay (Applied Biosystems Inc., Foster City, CA, USA) on an ABI 7500 real-time PCR (qPCR) System (Applied Biosystems) for detection of IL-10 rs1800871, rs1800872, rs1800896, and TNFa rs1799724. A $25 \mu \mathrm{L}$ PCR reaction mixture consisted of $1 \mathrm{X}$ TaqMan ${ }^{\circledR}$ Genotyping Master Mix (Applied Biosystems), 1X SNP Genotyping Assay Mix, and 50 ng DNA. Each 96-well plate included two no-template controls. qPCR was performed on an ABI 7500 system using the recommended conditions consisting of incubation at $95^{\circ} \mathrm{C}$ for $10 \mathrm{~min}$ followed by 40 cycles, denaturation at $92^{\circ} \mathrm{C}$ for $15 \mathrm{~s}$, and annealing/extension at $60^{\circ} \mathrm{C}$ for $1 \mathrm{~min}$. The VIC® and 6-carboxy-fluorescein (FAM) fluorescence levels of the PCR products were measured at $60^{\circ} \mathrm{C}$ for $1 \mathrm{~min}$. Analysis of fluorescence using the automated 2-color allele discrimination software on ABI 7500 showed clear discrimination of the two genotypes on a two-dimensional graph.

\section{Statistical analysis}

PCOS prevalence was estimated using sample proportion in order to see the relationship between the frequency in case and non-case groups, and for gene polymorphisms, the crude risk ratio (RR) and the crude odds ratio (OR) were used. A chi-squared test for dependency $(\chi 2)$ used to assess the frequencies of IL-10 and TNF $a$ gene polymorphisms. Logistic regression was used to examine the effects of gene polymorphisms on PCOS. A replicated stratification analysis was utilized in order to estimate the adjusted RR and adjusted OR to ensure that there was no confounders for age and body mass index (BMI). All statistical analyses were conducted using IBMSPSS (version 22).

\section{RESULTS}

\section{Demographic characteristics of patients and controls}

Clinical characteristics and statistics of PCOS patients and controls participating in this study are outlined in Table 1. The PCOS and the control groups had no significant differences in age.

Table 1. Demographic data of polycystic ovary syndrome (PCOS) patients and control subjects.

\begin{tabular}{lccc}
\hline Parameter & Control Mean \pm SEM & $\begin{array}{l}\text { PCOS } \\
\text { Mean } \pm \text { SEM }\end{array}$ & P value \\
\hline Age (yrs) & $31.48 \pm 0.489$ & $31.05 \pm 0.590$ & 0.572 \\
Height $(\mathrm{cm})$ & $158.35 \pm 0.628$ & $158.16 \pm 0.539$ & 0.817 \\
Weight $(\mathrm{kg})$ & $71.57 \pm 1.37$ & $69.25 \pm 1.25$ & 0.213 \\
BMI $(\mathrm{kg} / \mathrm{m} 2)$ & $28.54 \pm 0.522$ & $27.75 \pm 0.528$ & 0.290 \\
\hline
\end{tabular}

SEM= Standard Error of Mean; $\mathrm{P}=$ Significance 


\section{IL-10 gene (rs1800871) A/G polymorphism}

The prevalence of IL-10 polymorphisms was determined for Saudi women (98 PCOS patients and 93 controls). Table 2 presents the base pairs in the wild type homozygous (AA), heterozygous (AG), and mutated homozygous (GG). Overall, the distribution of the various genotypes of IL-10 (rs1800871) did not differ significantly between PCOS patients and controls $(\mathrm{P}=0.796)$.

Table2. IL-10 (rs1800871) A/G genotype in 98 polycystic ovary syndrome (PCOS) patients compared to 93 controls.

\begin{tabular}{|c|c|c|c|c|c|c|}
\hline \multirow{2}{*}{ Genotype } & \multirow{2}{*}{ Control No. } & \multirow{2}{*}{ PCOS No. } & \multicolumn{4}{|c|}{ Control vs. Patients } \\
\hline & & & OR & CI & $\chi^{2}$ & $P$ - value \\
\hline AA & 5 & 7 & 0.661 & $0.202-2.159$ & 0.48 & 0.490 \\
\hline AG & 45 & 36 & 0.571 & $0.167-1.952$ & 0.81 & 0.367 \\
\hline GG & 48 & 50 & 0.826 & $0.468-1.457$ & 0.44 & 0.508 \\
\hline TOTAL & 98 & 93 & & & & \\
\hline \multirow{2}{*}{ Allele } & Control & PCOS & \multicolumn{2}{|c|}{ Control vs. Patients } & & \\
\hline & (Freq) & (Freq) & OR & Cl & $\chi^{2}$ & P - value \\
\hline $\mathrm{A}$ & 0.287 & 0.261 & 1.061 & $0.677-1.663$ & 0.07 & 0.796 \\
\hline $\mathrm{G}$ & 0.738 & 0.712 & 0.943 & $0.601-1.478$ & & \\
\hline
\end{tabular}

No.: Number of individuals, OR: Odds Ratio, CI : Confidence Interval, $\chi^{2}$ : Chi Square

\section{IL-10 gene (rs1800872) T/G polymorphism}

The prevalence of the IL-10 polymorphism was determined for Saudi women (98 PCOS patients and 93 controls). Table 3 presents the base pairs in mutated homozygous (GG), heterozygous (TG), and also the wild type homozygous (TT). The overall, distribution of the various genotypes of IL-10 (rs1800872) did not differ significantly between PCOS and controls $(\mathrm{P}=0.693)$

Table3. IL-10 (rs1800872) T/G genotype in polycystic ovary syndrome (PCOS) patients compared to controls.

\begin{tabular}{|c|c|c|c|c|c|c|}
\hline \multirow{2}{*}{ Genotype } & \multirow{2}{*}{$\begin{array}{l}\text { Control } \\
\text { No. }\end{array}$} & \multirow{2}{*}{$\begin{array}{l}\text { PCOS } \\
\text { No. }\end{array}$} & \multicolumn{4}{|c|}{ Control vs. PCOS } \\
\hline & & & OR & CI & $\chi^{2}$ & $P$ - value \\
\hline TT & 5 & 5 & 0.946 & $0.265-3.381$ & 0.01 & 0.932 \\
\hline TG & 43 & 37 & 0.860 & $0.231-3.206$ & 0.05 & 0.822 \\
\hline GG & 50 & 51 & 0.858 & $0.486-1.515$ & 0.28 & 0.597 \\
\hline TOTAL & 98 & 93 & & & & \\
\hline Allele & $\begin{array}{l}\text { Control } \\
\text { (Freq) }\end{array}$ & $\begin{array}{l}\text { PCOS } \\
\text { (Freq) }\end{array}$ & $\begin{array}{l}\text { Contr } \\
\text { OR }\end{array}$ & $\mathrm{Cl}$ & $\chi^{2}$ & P - value \\
\hline $\mathrm{T}$ & 0.277 & 0.247 & 1.096 & $0.694-1.731$ & 0.16 & 0.693 \\
\hline $\mathrm{G}$ & 0.748 & 0.728 & 0.912 & $0.578-1.441$ & & \\
\hline
\end{tabular}

No.: Number of individuals, OR: Odds Ratio, CI : Confidence Interval, $\chi^{2}$ : Chi Square, Freq $=$ Frequency

\section{IL-10 gene (rs1800896) T/C polymorphism}


The prevalence of IL-10 polymorphism was determined for Saudi women (98 PCOS patients and 93 controls). Table 4 presents the frequencies of the wild type homozygous (TT), heterozygous (TC), and mutated homozygous (CC). Overall, the distribution of the various genotypes of IL-10 (rs1800896) did not differ significantly between PCOS patients and controls $(\mathrm{P}=0.141)$.

Table 4. IL-10 (rs1800896) T/C genotype in polycystic ovary syndrome (PCOS) patients compared to controls.

\begin{tabular}{|c|c|c|c|c|c|c|}
\hline \multirow{2}{*}{ Genotype } & \multirow{2}{*}{$\begin{array}{l}\text { Control } \\
\text { No. }\end{array}$} & \multirow{2}{*}{$\begin{array}{l}\text { PCOS } \\
\text { No. }\end{array}$} & \multicolumn{4}{|c|}{ Control vs. PCOS } \\
\hline & & & OR & CI & $\chi^{2}$ & p-value \\
\hline TT & 42 & 28 & 1.741 & $0.958-3.163$ & 3.34 & 0.067 \\
\hline $\mathrm{TC}$ & 38 & 46 & 1.816 & $0.955-3.453$ & 3.33 & 0.067 \\
\hline $\mathrm{CC}$ & 18 & 19 & 0.876 & $0.427-1.797$ & 0.13 & 0.718 \\
\hline TOTAL & 98 & 93 & & & & \\
\hline \multirow{2}{*}{ Allele } & Control & PCOS & \multicolumn{2}{|c|}{ Control vs. PCOS } & & \\
\hline & (Freq) & (Freq) & OR & Cl & $\chi^{2}$ & p-value \\
\hline $\mathrm{T}$ & 0.638 & 0.534 & 1.358 & $0.902-2.043$ & 2.16 & 0.141 \\
\hline $\mathrm{C}$ & 0.387 & 0.439 & 0.737 & $0.490-1.108$ & & \\
\hline
\end{tabular}

No.: Number of individuals, OR: Odds Ratio, CI : Confidence Interval, $\chi^{2}$ : Chi Square

\section{TNF-a gene (rs1799724) C/T polymorphism}

The prevalence of the IL-10 polymorphism was determined for Saudi women (98 PCOS patients and 93 controls). Table 5 presents the base pairs in the wild type homozygous (CC), heterozygous (CT), and mutated homozygous (TT). Overall, the distribution of the various genotypes of IL-10 (rs1799724) did not differ significantly between PCOS and control groups $(\mathrm{P}=0.953)$.

Table 5. TNF-a (rs1799724) C/T genotype in polycystic ovary syndrome (PCOS) compared to controls.

\begin{tabular}{|c|c|c|c|c|c|c|}
\hline \multirow{2}{*}{ Genotype } & \multirow{2}{*}{$\begin{array}{l}\text { Control } \\
\text { No. }\end{array}$} & \multirow{2}{*}{$\begin{array}{l}\text { PCOS } \\
\text { No. }\end{array}$} & \multicolumn{4}{|c|}{ Control vs. PCOS } \\
\hline & & & OR & CI & $\chi^{2}$ & $P$ - value \\
\hline $\mathrm{CC}$ & 85 & 80 & 1.062 & $0.465-2.430$ & 0.02 & 0.885 \\
\hline CT & 12 & 13 & 1.151 & $0.496-2.671$ & 0.11 & 0.743 \\
\hline $\mathrm{TT}$ & 1 & 0 & 2.877 & $0.116-71.513$ & 0.95 & 0.328 \\
\hline TOTAL & 98 & 93 & & & & \\
\hline \multirow{2}{*}{ Allele } & Control & PCOS & \multicolumn{2}{|c|}{ Control vs. PCOS } & & \\
\hline & (Freq) & (Freq) & OR & CI & $\chi^{2}$ & $P$ - value \\
\hline $\mathrm{C}$ & 0.952 & 0.905 & 0.977 & $0.446-2.138$ & \multirow{2}{*}{0.00} & \multirow[t]{2}{*}{0.953} \\
\hline $\mathrm{T}$ & 0.073 & 0.068 & 1.024 & $0.468-2.240$ & & \\
\hline
\end{tabular}

No.: Number of individuals, OR: Odds Ratio, CI : Confidence Interval, $\chi^{2}$ : Chi Square

\section{DISCUSSION}

Lymphocytes and monocytes produce IL-10 that has been shown to be an essential anti-inflammatory compound and immune-balancing cytokine among others because it efficiently down-regulates pro-inflammatory cytokines, such as IL-1 and -6 and TNF $\alpha$. Since an imbalance in pro- and anti-inflammatory cytokines could participate in PCOS, the 
polymorphic sequence of IL-10 may be a crucial biomarker for PCOS predisposition (Karadeniz et al., 2008; Talaat et al., 2016).

Our results were compared with those reported in literature and showed that our findings were in agreement with those of several studies that indicated that numerical combinations of genetic polymorphism of IL-10 do not influence PCOS (Karadeniz et al., 2008; Vural et al., 2010; Lindholm et al., 2011; Sóter et al., 2015). Results by Talaat et al. (2016) did not agree with our findings in a study in 80 controls in relation to 61 patients with PCOS in Egyptian women. They examined the influence of genetic polymorphisms in addition to IL-10 serum levels as risks for PCOS. Results showed that IL-10 -1082 GG and -819 TT genotype could be considered one of the causes for PCOS, and IL-10 levels in normal controls were higher compared to PCOS patients. A study by Xing et al. (2017) reported an association between growth of PCOS in the Chinese population and the IL-10 rs1800871 polymorphism.

Numeral ailments are regulated by $\mathrm{TNF} \alpha$, which is a multifunctional proinflammation cytokine. It exists not only in oocytes and granulose cells, but also in human ovarian follicular fluid. TNF $\alpha$ is believed to be connected with anovulation, increased ovarian steroid secretion, and ovarian apoptosis (Yun et al., 2011).

The results for the TNFa (rs1799724) $\mathrm{C} / \mathrm{T}$ polymorphism also showed that the majority of female patients and controls were homozygous for $\mathrm{CC}$, and about $12 \%$ of the controls and $14.0 \%$ of PCOS patients existed in the heterozygous (CT) state and also in the wild type allele (CC). Nevertheless, PCOS and control groups did were not significantly different.

Comparison of our results with those reported in literature indicated that, our analysis agreed with one study in which it was inferred that numeral combinations of genetic polymorphisms of TNF $\alpha$ had no relationship with PCOS (Vural et al., 2010; Gou et al., 2015). On the other hand, there is a contrasting study by Yun et al. (2011) in which 217 PCOS patients and 144 matched female controls (healthy women) were studied. A comparative study of the $-1031(\mathrm{~T} / \mathrm{C})$ polymorphism of the TNF $\alpha$ gene with PCOS in a Korean population was done, and the outcome showed that there was a strong relationship between PCOS $(\mathrm{P}-$ value $=0.0003, \mathrm{OR}=2.53)$ and the $-1031(\mathrm{~T} / \mathrm{C})$ polymorphism in the promoter region of the TNF $\alpha$ gene. Moreover, the occurrence of the $\mathrm{C}$ allele was lower in controls in comparison to PCOS patients. Thathapudi et al. (2014) reported that the TNF $\alpha$ system might contribute to the pathogenesis of $\mathrm{HA}, \mathrm{Ob}$, and IR in PCOS patients independent of the polymorphism of the TNF- $\alpha$ C850T (rs1799724) as shown in an Indian population.

However, our results do not mean that a relationship between SNPs and PCOS does not exist; differences may be due to differences in life styles. There are no reports on the genetic polymorphisms in cytokine genes in Saudi PCOS patients, and we need more research concerning cytokines associated with PCOS, especially in the Saudi population whose women are the most vulnerable.

\section{ACKNOWLEDGMENTS}

The authors would like to thank the Deanship of Scientific Research at King Saud University for funding this study through the Research Project. 


\title{
CONFLICTS OF INTEREST
}

\author{
The authors declare no conflict of interest.
}

\section{REFERENCES}

Couper KN, Blount DG and Riley EM (2008). IL-10: the master regulator of immunity to infection. J. Immunol. 180: 5771-5777.

Fulghesu AM, Sanna F, Uda S, Magnini R, et al. (2011). IL-6 serum levels and production is related to an altered immune response in polycystic ovary syndrome girls with insulin resistance. Mediators Inflamm. 2011: 389317

Guo R, Zheng Y, Yang J and Zheng N (2015). Association of TNF-alpha, IL-6 and IL-1beta gene polymorphisms with polycystic ovary syndrome: a meta-analysis. BMC Genet. 16: 5 .

Karadeniz M, Erdogan M, Zengi A, Tamsel S, et al. (2008). Polymorphism of the interleukin-10 gene in polycystic ovary syndrome. Int. J. Immunogenet. 35: 119-123.

Lindholm Å, Blomquist C, Bixo M, Dahlbom I, et al. (2011). No difference in markers of adipose tissue inflammation between overweight women with polycystic ovary syndrome and weight-matched controls. Hum Reprod. 26: 14781485.

Liu F, Dai W, Li C, Lu X, et al. (2016). Role of IL-10-producing regulatory B cells in modulating T-helper cell immune responses during silica-induced lung inflammation and fibrosis. Sci. Rep. 6: 28911.

Qin L, Xu W, Li X, Meng W, et al. (2016). Differential expression profile of immunological cytokines in local ovary in patients with polycystic ovarian syndrome: analysis by flow cytometry. Eur J Obstet Gynecol Reprod Biol. 197: 136-141.

Sóter MO, Ferreira CN, Sales MF, Candido AL, et al. (2015). Peripheral blood-derived cytokine gene polymorphisms and metabolic profile in women with polycystic ovary syndrome. Cytokine. 76: 227-235.

Szczuko M, Zapałowska-Chwyć M, Drozd A, Maciejewska D, et al. (2016). Effect of IGF-I and TNF- $\alpha$ on intensification of steroid pathways in women with PCOS phenotypes are not identical. Enhancement of progesterone pathway in women with PCOS increases the concentration of TNF- $\alpha$. Gynecol Endocrinol. 32: 714717.

Talaat RM, Mohamed YA, Mohamad EH, Elsharkawy M, et al. (2016). Interleukin 10 (-1082 G/A) and (- 819 C/T) gene polymorphisms in Egyptian women with polycystic ovary syndrome (PCOS). Meta gene. 9: 254-258.

Thathapudi S, Kodati V, Erukkambattu J, Katragadda A, et al. (2014). Tumor necrosis factor-alpha and polycystic ovarian syndrome: a clinical, biochemical, and molecular genetic study. Genet. Test Mol. Biomarkers. 18: 605-609.

Vural P, Değirmencioğlu S, Saral NY and Akgül C (2010). Tumor necrosis factor $\alpha(-308)$, interleukin-6 (-174) and interleukin-10 (-1082) gene polymorphisms in polycystic ovary syndrome. Eur. J. Obstet. Gynecol. Reprod. Biol. 150: 61-65.

Wang L, Qi H, Baker PN, Zhen Q, et al. (2017). Altered circulating inflammatory cytokines are associated with anovulatory polycystic ovary syndrome (PCOS) women resistant to clomiphene citrate treatment. Med. Sci. Monit. 23: 1083.

Wu R, Fujii S, Ryan NK., Van der Hoek KH, et al. (2007). Ovarian leukocyte distribution and cytokine/chemokine mRNA expression in follicular fluid cells in women with polycystic ovary syndrome. Hum. Reprod. 22: 527-535.

Xing G, Sun Y, Wang F, Gao G, et al. (2017). Role of interleukin-10 polymorphisms and haplotypes in polycystic ovary syndrome risk. Int. J. ClinExp Pathol. 10: 7956-7961.

Yamada-Nomoto K, Yoshino O, Akiyama I, Iwase A, et al. (2017). PAI-1 in granulosa cells is suppressed directly by statin and indirectly by suppressing TGF- $\beta$ and TNF- $\alpha$ in mononuclear cells by insulin-sensitizing drugs. Am. $J$. Reprod. Immunol. 78: e12669.

Yun JH, Choi JW, Lee KJ, Shin JS, et al. (2011). The promoter-1031 (T/C) polymorphism in tumor necrosis factor-alpha associated with polycystic ovary syndrome. Reprod. Biol. Endocrinol. 9: 131.

Zeng B, Lai Z, Sun L, Zhang Z, et al. (2018). Structural and functional profiles of the gut microbial community in polycystic ovary syndrome with insulin resistance (IR-PCOS): a pilot study. Res. Microbiol. 170: 43-52.

Zhang H, Bi Y, Hu C, Lu W, et al. (2012). Association between the Pro12Ala polymorphism of PPAR- $\gamma$ gene and the polycystic ovary syndrome: A meta-analysis of case-control studies. Gene. 503: 12-17.

Zhang T, Tian F, Huo R, Tang A, et al. (2017). Detection of dendritic cells and related cytokines in follicular fluid of patients with polycystic ovary syndrome. Am. J. Reprod. Immunol. 78: e12717.

Zhang L, Fang X, Li L, Liu R, et al. (2018). The association between circulating irisin levels and different phenotypes of polycystic ovary syndrome. J. Endocrinol. Invest. 41:1401-1407.

Zhang JM and An J (2007). Cytokines, inflammation and pain. Int. Anesthesiol. Clin. 45: 27-37. 